\title{
Resultados perinatales en el síndrome de transfusión feto fetal manejados con la técnica de Solomon. Revisión de la literatura
}

\author{
Saulo Molina G. MSc ${ }^{1}$, Rodolfo Casas $P^{1}{ }^{1}$, Roberth Ortiz M. MSc ${ }^{1}$ \\ ${ }^{1}$ Unidad de Terapia, Cirugía Fetal y Fetoscopia, División de Medicina Materno fetal, Departamento de Obstetricia y \\ Ginecología, Hospital de San José; Unidad de Terapia y Cirugía Fetal Clínica Colsubsidio Materno Infantil y Departamento \\ de Ginecología y Obstetricia de la Fundación Universitaria de Ciencias de la Salud - FUCS. Bogotá, Colombia.
}

\section{RESUMEN}

Introducción: Entre 15-20\% de los embarazos gemelares monocoriales biamnióticos se complican con el síndrome de transfusión feto/fetal el cual se asocia con mortalidad superior a $90 \%$ y morbilidad significativa en el $50 \%$ del gemelo sobreviviente. La técnica láser que coagula la superficie de la placa coriónica entre los principales canales a lo largo del ecuador (técnica de Solomon), se ha sugerido para disminuir la recurrencia, prevenir complicaciones secundarias sin incrementar resultados adversos. Métodos: Revisión de la literatura existente en las bases de datos MEDLINE, EBSCO, OVID, PROQUEST, COCHRANE, Lilacs, SciELO, desde el año 2000 al 2015. Se incluyeron los artículos de revisión e investigaciones originales que compararon la técnica estándar de fotocoagulación secuencial con láser y la técnica de Solomon, el resultado primario fue la reducción de la incidencia Secuencia Anemia Policitemia, recurrencia del síndrome de transfusión feto/fetal, mortalidad perinatal y morbilidad neonatal severa. Resultados: Se encontraron 200 artículos, se seleccionaron seis: 1 ensayo clínico y su análisis secundario, 2 estudios de cohorte retrospectivos, 1 revisión sistemática y un estudio que compara los resultados del neurodesarrollo. Los estudios sugieren una mejoría en la sobrevida de algunos de los fetos con la técnica Solomon, menor recurrencia del síndrome de transfusión feto/fetal y Secuencia Anemia Policitemia, sin la presencia de eventos adversos. Conclusión: La técnica de Solomon mejora la sobrevida de algunos gemelos, sin embargo no puede concluirse que haya mejoría en la mortalidad pues los estudios no tienen el suficiente poder para determinarlo.

\section{PALABRAS CLAVE: Cirugía láser, fetoscopia, gemelos monocoriónicos, síndrome de transfusión feto fetal, secuencia anemia-policitemia, secuencia oligo-polihidramnios}

\section{SUMMARY}

Introduction: Between 15 to $20 \%$ of monochorionic diamniotic twin pregnancies are complicated by the twin-twin transfusion syndrome. It has a mortality greater than $90 \%$ and a significant morbidity, $50 \%$ in the surviving twin. The Solomon technique (laser photocoagulation of the main vascular channels of the chorionic plate surface along the entire vascular equator) has been suggested to reduce the recurrence, and prevent secondary complications without increasing adverse results. Methods: Systematic review of electronic searches of the literature from 2000 to 2015 (MEDLINE, EBSCO, OVID, PROQUEST, COCHRANE, Lilacs, and SciELO). We included review articles and original investigations comparing the standard photocoagulation technique with laser ablation against the Solomon technique. The primary results were reduction of Anemia Polycythemia Sequence incidence, twin-twin transfusion syndrome recurrence, perinatal mortality and severe neonatal morbidity. Results: Of 200 articles, we selected six: one clinical essay and its secondary analysis, two retrospective cohort studies, one systematic review and a study comparing neurodeve- 
lopmental outcomes. The studies suggested a survival improvement in some fetuses using the Solomon technique, less twin-twin transfusion syndrome recurrence and Anemia Polycythemia Sequence without the presence of adverse effects. Conclusion: Solomon technique improves the survival of some twins, although we cannot conclude there is mortality improvement, because the studies do not have enough power to determine that.

\section{KEY WORDS: Laser surgery, fetoscopy, monochorionic twins, twin-twin transfusion syndrome, twin anemia-polycithemia sequence, twin oligo-polyhydramnios sequence}

\section{INTRODUCCIÓN}

El embarazo gemelar monocorial-biamniótico representa el $15 \%$ de los embarazos gemelares espontáneos y cerca del $5 \%$ de embarazos gemelares asistidos médicamente. Aproximadamente 15-20\% se complican con el síndrome de transfusión feto/ fetal $(1,2)$. Una red de transfusión de flujo desequilibrado de sangre desde un feto (donante) a la otra (receptor) a través de las anastomosis vasculares placentarias se ha propuesto como etiología $(2,3)$, aunque la documentación del desequilibrio de flujo ha sido difícil de establecer (4).

Debido a la baja la resistencia vascular, son posibles grandes cambios de volumen de sangre a través de anastomosis arterio-arteriales que por un lado permite la compensación de los cambios de volumen que se producen a través de la anastomosis arterio-venosa predominantemente en una dirección, y por otro lado, facilita un cambio de sangre hacia un co-gemelo eventualmente hipotensor, con la consecuente hipovolemia y el riesgo de encefalopatía hipóxico-isquémica en el gemelo normotenso (5). Tales accidentes vasculares inter gemelos junto con la transfusión crónica a través de anastomosis arterio-venosas, se cree son responsables del aumento de la mortalidad y la morbilidad en los pares de gemelos monocorial-biamniótico (6). De no tratarse este síndrome se asocia con tasas de mortalidad altas ( $90 \%$ ) y morbilidad significativa del $50 \%$ del gemelo sobreviviente, específicamente secuelas neurológicas muchas veces irreversibles $(1,2)$. En caso de fallecimiento intrauterino de uno de los gemelos, el sobreviviente tiene riesgo entre 30 a $35 \%$ de muerte intrauterina consecutiva (7) y riesgo de lesión neurológica del $26 \%$ (2\% en gemelos bicoriales). En general, los gemelos monocorial biamnióticos son 4,81 veces más propensos a tener morbilidad del neurodesarrollo (8).

La amniorreducción seriada es eficaz en la prolongación del embarazo mediante la reducción de la cantidad de líquido amniótico para evitar la ruptura prematura de las membranas y el trabajo de parto pretérmino (8), pero la visualización fetoscópica y la ablación laser de las anastomosis placentarias se establece como la terapia curativa de primera línea $(8,9)$ (Figuras 1 y 2). Inicialmente se llevó a cabo la coagulación laser no selectiva de los vasos placentarios $(8,10,11)$, se informó posteriormente la identificación y coagulación laser selectiva de los vasos placentarios en cada anastomosis que cruzan entre gemelos (ecuador vascular) (12).

Una técnica láser que coagula la superficie de la placa coriónica entre los principales canales a lo largo del ecuador vascular ha sido descrita; esta dicorionización ecuatorial o técnica de Solomon, descrita primero por el grupo de Leiden en los Países Bajos (13), se ha sugerido para disminuir la recurrencia. Coagula una línea a través del ecuador vascular de la superficie coriónica después de la ablación selectiva, separando los territorios vasculares de cada gemelo, reduciendo las complicaciones, sin aumentar los resultados adversos $(14,15)$ (Figuras 3 y 4). Estudios contemporáneos (16) reportan incremento de la tasa de supervivencia de al menos uno de los gemelos del 70 al $88 \%$ en los últimos 25 años, $y$ del 35 al $65 \%$ para ambos gemelos $(p=0,012)$ y por lo menos un gemelo del 70 al $88 \%(p=0,009)$ mejorando significativamente la sobrevida perinatal con la evolución de la técnica laser de no selectiva a selectiva, selectiva secuencial y técnica de Solomon $(p=0,01)$. El parto pretérmino continúa siendo un desafío ya que determina la sobrevida y morbilidad perinatal $(2,4)$. La edad gestacional promedio en el parto varía entre 29 a 33 semanas con una amplia variación entre centros $(5,14)$.

Toda nueva intervención en salud debe ser evaluada, por lo que se planteó la presente revisión en la literatura, con el objetivo de evaluar la evidencia que soporta la eficacia y seguridad de esta nueva técnica de Solomon vs la estándar, respecto a resultados en sobrevida de los fetos, complicaciones a largo plazo como las neurológicas, de la misma manera la necesidad de re-intervenciones por reaparición del síndrome de transfusión feto/fetal o por aparición de secuencia anemia policitemia. 


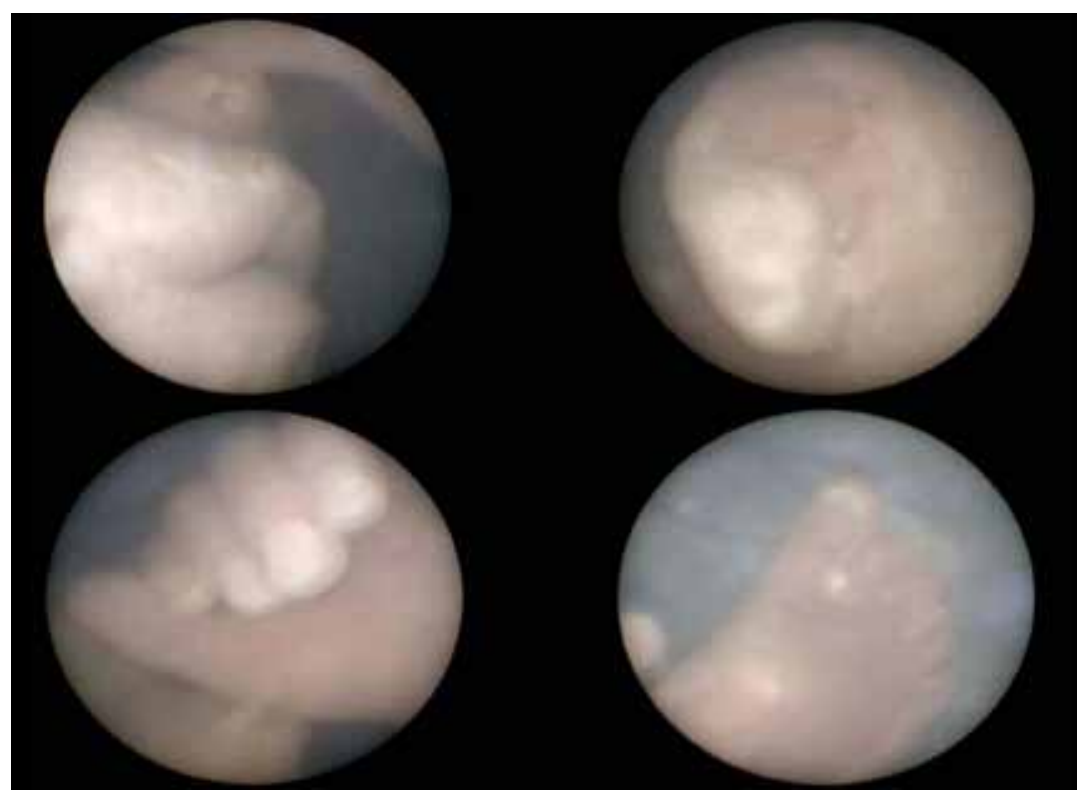

Figura 1. Fetoscopia operatoria. Valoración anatómica fetal. Se observa labio, oreja, mano y pie del feto.

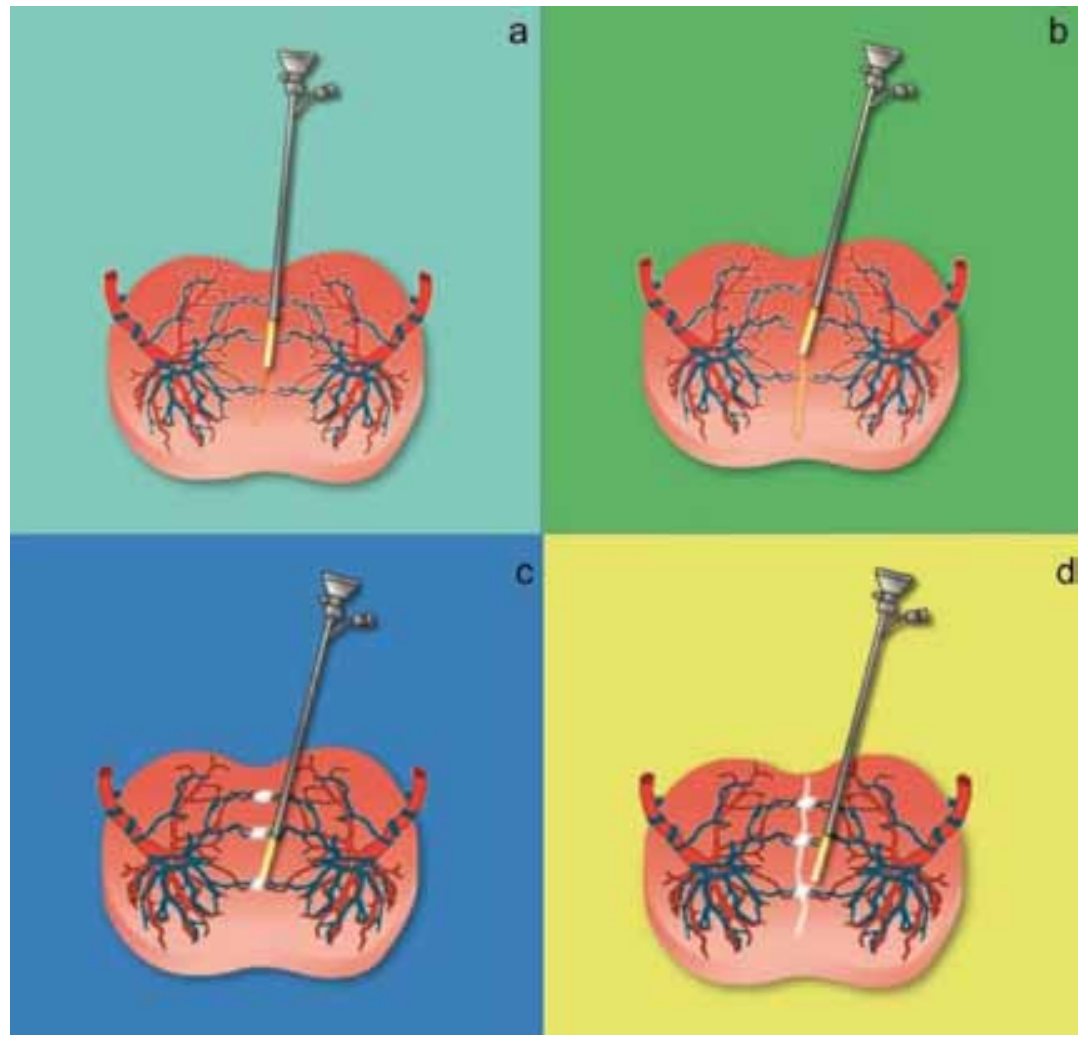

Figura 2. Diferentes técnicas reportadas para ablación de vasos comunicantes: a y b muestran dicotomizacion de ecuador vascular placentario. c. Técnica ultraselectiva. d. Técnica de Solomon o solomonización del ecuador vascular placentario. 

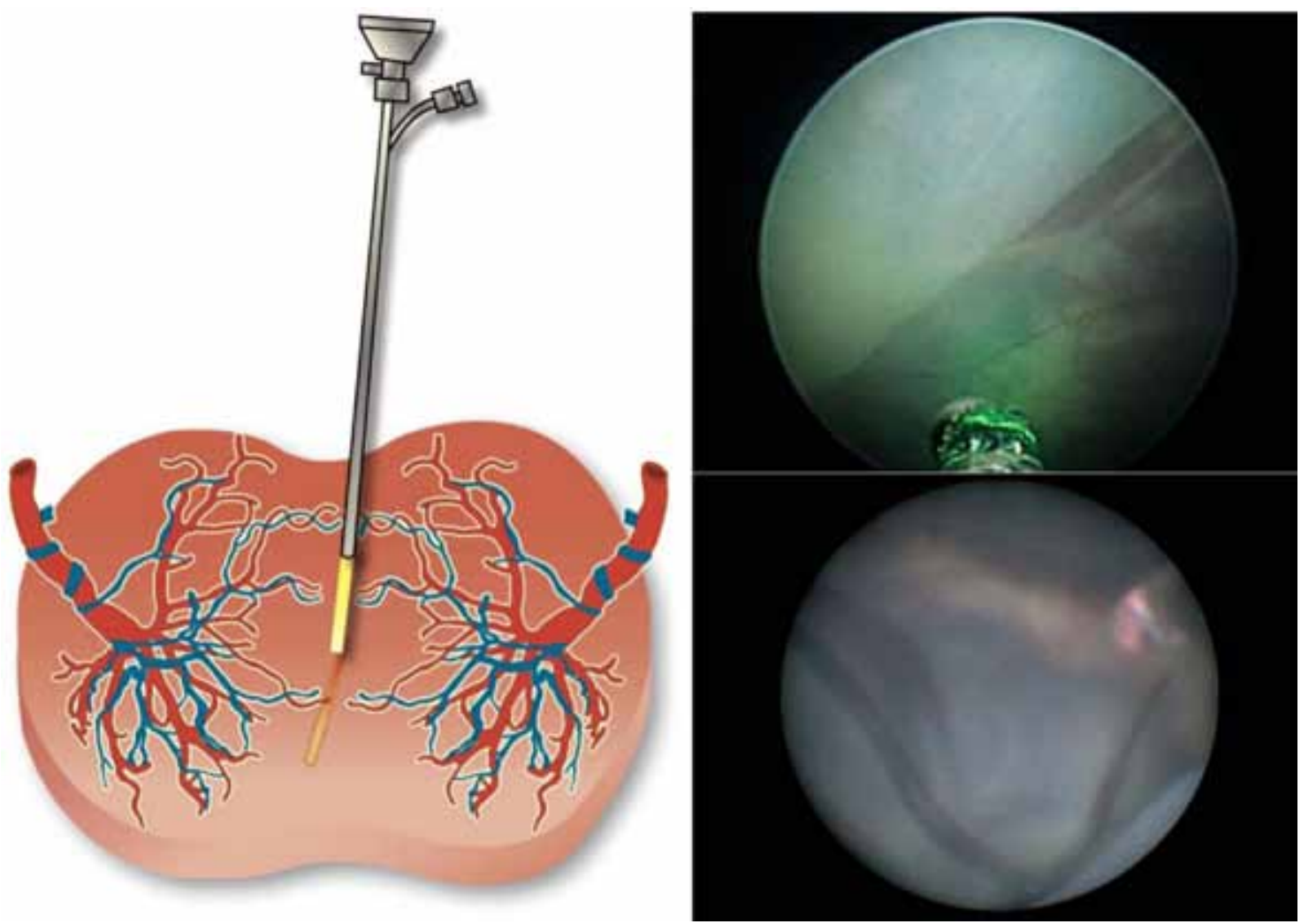

Figura 3. Esquema muestra anastomosis vasculares con fulguración de las mismas a nivel del ecuador placentario. A la derecha superior identificación de ecuador placentario con vasos comunicantes. Derecha inferior solomonización.

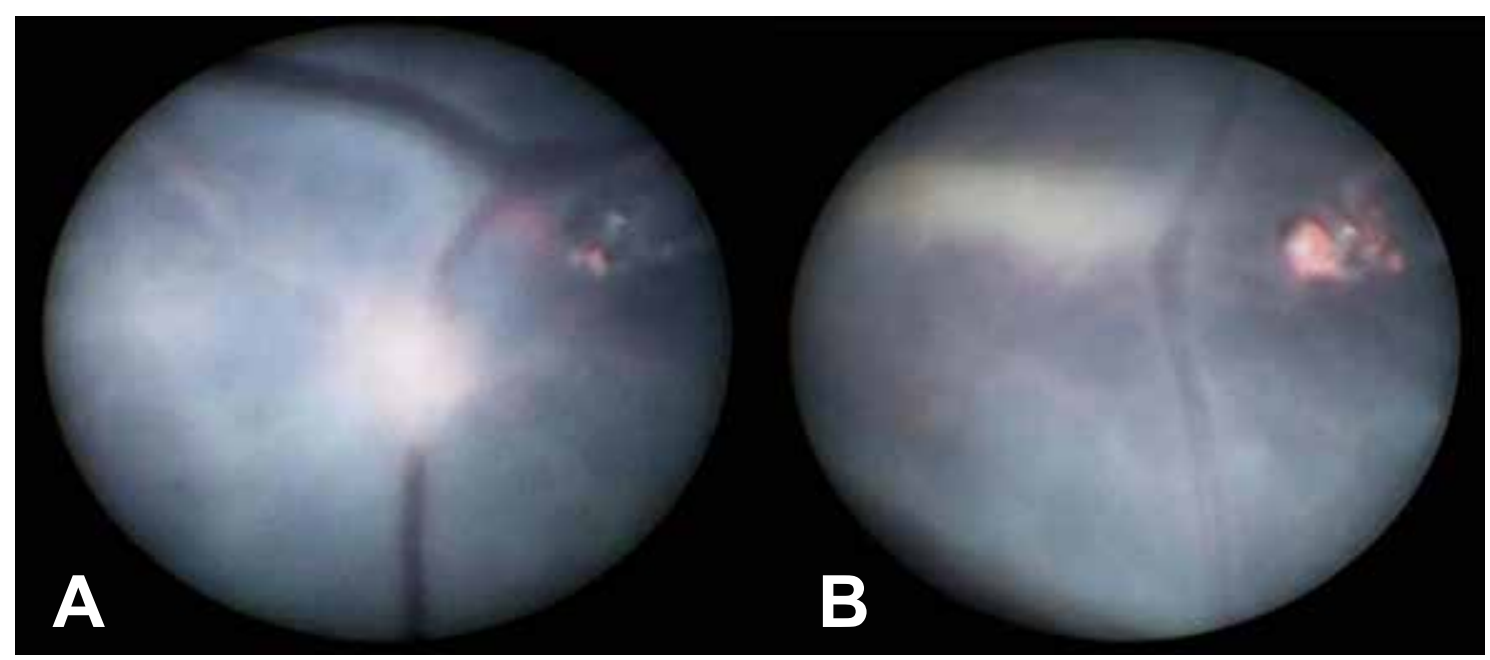

Figura 4. A: muestra fulguración de vasos comunicantes placentarios. B: solomonización. 


\section{MATERIALES Y METODOS}

Se realizó una búsqueda en la literatura mundial en diferentes bases de datos, MEDLINE, EBSCO, OVID, PROQUEST, COCHRANE, y SciELO. Los artículos buscados fueron tanto en español como en inglés desde el año 2000 al 2015. Se utilizaron términos MeSH "Fetoscopic laser surgery", "Monochorionic twins", "Twin-twin transfusión síndrome", "twin anemia-polycithemia sequence", "twin oligo polyhydramnios sequence". Los criterios de inclusión se formularon de acuerdo a nuestra pregunta preestablecida (PICO), que hace referencia a Paciente o Población, Intervención, Comparación, Resultado (Outcome). La población se refiere al embarazo gemelar monocorial biamniótico complicado por síndrome de transfusión feto/fetal diagnosticado usando criterios ecográficos prenatales estándar (17). La intervención es la técnica estándar de fotocoagulación secuencial con láser. La comparación de la técnica de Solomon y el resultado primario fue la incidencia de secuencia anemia policitemia, recurrencia del síndrome de transfusión feto/fetal, mortalidad perinatal o morbilidad neonatal severa. La calidad de los estudios fue evaluada por Consort para los ensayos clínicos (18), para la evaluación del riesgo de sesgos en estos se utilizó la herramienta Cochrane (19), para los estudios tipo cohorte se utilizó Strobe (20) y Prisma para las revisiones sistemáticas (21).

\section{RESULTADOS}

Después de la búsqueda por las diferentes bases de datos en la literatura teniendo en cuenta los términos MeSH referentes, se encontraron 200 artículos de los cuales la mayor parte hacen referencia a la comparación entre amnioreducción y fotocoagulación laser estándar; solo se encontró un ensayo clínico que cumplía con el criterio de inclusión: comparación directa entre la técnica estándar y la técnica de Solomon (22). Un análisis secundario del ensayo clínico ya mencionado cuya meta fue evaluar la ocurrencia y las características de las anastomosis residuales en las placentas (23); se encontraron además dos estudios tipo cohorte retrospectivos $(24,25)$ y una revisión sistemática, que incluyó el ensayo clínico y los dos estudios cohorte mencionados previamente (26) y un estudio que compara las dos técnicas según los resultados del neurodesarrollo (27), con base en estos estudios se realizaron los análisis.

El estudio de Ruano y cols (24), es observacional analítico tipo cohorte retrospectiva, realizado en 4 centros de referencia regional de España, Brasil y Estados Unidos, teniendo como muestra final 102 embarazos en un periodo de 2 años, los cuales fueron catalogados como síndrome de transfusión feto/fetal que ameritaban manejo con coagulación con láser, estado de Quintero II-IV; ventajas del estudio: procedimiento realizado por expertos, definición estandarizada del síndrome (ecográfica y clasificación de Quintero), descripción de las técnicas en forma clara, manejo post intervención según protocolos estandarizados (amniorreducción en todos los casos), resultados de interés bien definidos y estandarizados (sobrevida a los 30 días de uno o los dos gemelos, secuencia anemia policitemia y recurrencia de síndrome de transfusión feto/fetal). El análisis y metodología utilizados son acordes, se describen las diferentes variables y su fuente, se realizó regresión logística y modelos lineales generalizados mixtos lo que permitió el manejo y ajuste de posibles confusores; por todos estos motivos se califica como de buena calidad. Resultados: las características basales entre los dos grupos fueron comparables, la sobrevida a los 30 días es mejor para aquellos manejados con la técnica de Solomon, con una sobrevida para ambos gemelos en la técnica de Solomon de $84,6 \%$ y para la estándar de $46,1 \%$, lo cual fue estadísticamente significativo $(p<0,01)$. Con referencia a que sobreviviera al menos uno de ellos no se presentó diferencia estadística: $88,5 \%$ para técnica de Solomon y para su contraparte $77,6 \%(p=0,27)$. En el multivariado después de ajustar por edad gestacional de realización del procedimiento, centro de tratamiento, estado Quintero, la técnica de Solomon permaneció no relacionada con la probabilidad se sobrevida de al menos uno de los gemelos (ORa 2.01; IC95\% 0,4810,$75 ; p=0,37$ ), lo único significativo fue el estado Quintero II vs estados más avanzados (ORa 4,16; IC95\% 1,29-15,72: $p=0,02)$. En cuanto a la sobrevida de ambos gemelos en el multivariado (técnica de Solomon, localización de placenta, estado Quintero, centro de tratamiento) se encontró que la técnica de Solomon fue asociada con incremento de la sobrevida de ambos (ORa 11,35; IC95\% 3,11$53,14 ; p=0,0007)$. Para la sobrevida global, la técnica de Solomon presentó una sobrevida más alta y significativa (ORa IC95\% 1,59-13,62; $p=0,005$ ). Para la recurrencia de síndrome de transfusión feto/fetal y secuencia anemia policitemia no se presentaron casos en la técnica de Solomon y en la estándar se presentaron 5,3\% y 7,9\% respectivamente, pero estos hallazgos no fueron significativos $(p=0,57$ y $p=0,33$ respectivamente).

Dhillon y cols (26) encuentran evidencia que sugiere mejor sobrevida global con la técnica de Solomon, estadísticamente significativa para la sobrevida de al menos uno de los gemelos, tendencia hacia la reducción de la secuencia anemia policitemia, sin incremento de complicaciones o efectos adversos cuando se usó la técnica de Solomon comparada con la técnica selectiva.

En el análisis secundario del ensayo clínico previo (27), sobre las anastomosis residuales visualizadas en la pieza anatómica (placenta) la cual se 
realizó por una técnica estándar de tinción, de 151 placentas incluidas en el estudio, al comparar el porcentaje de anastomosis residuales en el grupo de Solomon se presentó en un $19 \%(14 / 74)$ y en la técnica estándar en un $34 \%(26 / 77)(p<0,05)$; hallazgo a tenerse en cuenta en el contexto de que la meta de la terapia laser es coagular todas las anastomosis vasculares y de esta manera prevenir complicaciones secundarias a éstas.

El estudio observacional analítico de Baschat (25) publicado en 2013, tipo cohorte retrospectiva, con datos recogidos entre el 2005 al 2011, tiene ventajas similares al descrito previamente, con diferencia en la definición de sobrevida a 6 meses. En los resultados se pueden evidenciar diferencias en las características basales: mayor número de afroamericanas y de índice de masa corporal, uso de técnica de reproducción asistida en el grupo de técnica no estándar; respecto a variables como edad gestacional al diagnóstico, peso estimado fetal, volumen de líquido amniótico, localización de placenta y estado Quintero fueron similares $(p>0,05)$. El análisis se realizó con 147 gemelos, 71 para laser estándar y 76 para TS; la sobrevida a los 6 meses fue significantemente más alta para la técnica de Solomon $68,4 \%$ vs 50,7\% ( $p<0,05)$. Esta fue asociada en forma significativa a una más baja recurrencia del síndrome de transfusión feto/fetal $3,9 \%$ vs $8,5 \%(p<0,05)$, Secuencia anemia policitemia $2,6 \%$ vs $4,2 \%(p<0,05)$ y anormalidades del líquido amniótico $0 \%$ vs $5,6 \%(p<0,05)$.

De estos dos estudios se pueden inferir que la técnica de Solomon al parecer presenta mejores tasa de sobrevida. Con respecto a la recurrencia de síndrome de transfusión feto/fetal y secuencia anemia policitemia el estudio de Ruano (24) no mostró diferencias, puede ser por la pobre potencia debido al tamaño de muestra, mientras que el estudio de Baschat (25) si mostró diferencias significativas respecto a estos dos puntos.

El tercer estudio de Slaghekke y cols (22), es un ensayo clínico controlado aleatorizado multicéntrico, abierto en 5 centros de referencia terciarios europeos. Tanto los criterios de inclusión y exclusión fueron claramente definidos, el resultado primario está bien descrito, compuesto de la incidencia de secuencia anemia policitemia, recurrencia de síndrome de transfusión feto/fetal, mortalidad perinatal o morbilidad neonatal severa, al igual que los secundarios, el tamaño de muestra es adecuado e incluso se ajustó posteriormente por efectos adversos, lo cual le da mayor confiabilidad. Al evaluar los sesgos se encontró una baja probabilidad de éstos en todos los dominios (19); la generación de la secuencia se realizó en forma adecuada por bloques permutados y estratificada por centro, la ocultación de la secuencia fue dada por un sistema en línea no accesible a los reclutadores; respecto del cegamiento, cabe anotar que no es posible cegar una técnica quirúrgica, pero a favor del estudio está que el protocolo para diagnósticos del resultado primario y los secundarios está estandarizado, los procedimientos fueron hechos por expertos y con instrumentos similares, las técnicas están descritas en forma clara al igual que los protocolos, se describen en forma completa todos los datos y resultados y se hizo por intensión a tratar, calificando este estudio como de buena calidad.

Se evaluaron 274 gestantes, 139 en la técnica de Solomon y 135 en la estándar, los grupos son comparables en sus características basales; al evaluar el resultado primario, este se presentó en un $34 \%$ (94/274 fetos) en la técnica de Solomon vs $49 \%$ (133/270 fetos) en la técnica estándar (OR $0,54$; IC $95 \% 0,35-0,82)$, lo que implica que hay una disminución de un $46 \%$ de este resultado con la técnica de Solomon, hallazgo significante al no cruzar la unidad. Al desglosar el resultado compuesto se encontró un OR 1,04; IC95\% 0,66-1,63 para sobrevida global al mes, OR 0,85 IC95 0,43-1,68 para al menos un sobreviviente y OR 1,16 IC95\% 0,71-1,89 para ambos sobrevivientes, hallazgos no significativos; sin embargo, para recurrencia del síndrome de transfusión feto/fetal se encontró un OR 0,21 IC95\% 0,04-0,98; para secuencia anemia policitemia un OR 0,16; IC95\% 0,05-0,49 ambos resultados con significancia estadística a favor de la técnica de Solomon. En cuanto a la morbilidad neonatal severa no se encontraron diferencias estadísticas entre ambos grupos (OR 0,65; IC95\% $0,31-1,36)$, para las complicaciones relacionadas con los procedimientos, tampoco se presentaron diferencias estadísticas significativas, todos los IC al $95 \%$ cruzaron la unidad; no se evidenciaron diferencias respecto del tiempo del procedimiento $(p>0,05)$. Con este estudio se puede sugerir que la técnica de Solomon reduce los riesgos de complicaciones asociadas con anastomosis residuales en el embarazo monocorial-biamniótico tratados con cirugía láser para síndrome de transfusión feto/fetal (Tabla I).

Van Klink y cols (28), publican un estudio de adecuada calidad cuyo objetivo fue comparar los resultados en el neurodesarrollo a largo plazo de los sobrevivientes incluidos en el estudio de Slaghekke y cols (22), encontrando sobrevivientes sin alteraciones en el $67 \%$ del grupo sometido a la técnica de Solomon vs $68 \%$ de la técnica estándar $(p=0,92)$, al comparar las alteraciones en el seguimiento del neurodesarrollo de los sobrevivientes no se encontró diferencia significativas entre los dos grupos (11\% técnica Solomón vs $9 \%$ técnica estándar; $p=0,61)$, en el desarrollo motor tampoco hubo diferencias significativas $(p=0,23)$. Los autores concluyen que no existen diferencias estadísticamente significativas en el neurodesarrollo de los sobrevivientes de ambos grupos (Tabla II). 
Tabla I

SÍNDROME DE TRANSFUSIÓN FETO FETAL, RESULTADOS DEL SEGUIMIENTO: TÉCNICA CONVENCIONAL VS TÉCNICA DE SOLOMON

\begin{tabular}{|c|c|c|c|c|c|c|}
\hline Estudio & $\begin{array}{l}\text { Sobrevida } \\
\text { al menos un } \\
\text { gemelo }\end{array}$ & $\begin{array}{c}\text { Sobrevida } \\
\text { ambos gemelos }\end{array}$ & $\begin{array}{c}\text { TAPS (Antenatal } \\
\text { o posnatal) }\end{array}$ & $\begin{array}{l}\text { Recurrencia } \\
\text { STFF }\end{array}$ & Infección & RPMO \\
\hline \multirow[t]{3}{*}{ Slaghekke y cols ${ }^{23}$} & 1 mes post parto & 1 mes post parto & TC:15,6\% & TC:6,7\% & TC:0\% & TC:34,1\% \\
\hline & TC:86,7\% & TC:60,0\% & TS:2,9\% & TS:1,5\% & TS:0,7\% & TS:41,6\% \\
\hline & TS:84,7\% & TS:63,5\% & & & $\begin{array}{l}\text { OR } 0,34 \\
(\text { IC95\% } \\
0,002-6,35)\end{array}$ & \\
\hline \multirow[t]{3}{*}{ Ruano y cols ${ }^{24}$} & 1 mes post parto & 1 mes post parto & TC:7.0\% & TC:5,3\% & - & - \\
\hline & TC:77,6\% & TC: $46,1 \%$ & TS:0\% & TS:0\% & & \\
\hline & TS:88,5\% & TS:84,6\% & & & & \\
\hline \multirow[t]{3}{*}{ Baschat y cols ${ }^{25}$} & $\begin{array}{l}6 \text { meses post } \\
\text { parto }\end{array}$ & $\begin{array}{l}6 \text { meses post } \\
\text { parto }\end{array}$ & TC:4,2\% & TC:8,5\% & TC:1,4\% & TC:2,8\% \\
\hline & TC:98\% & TC:76\% & TS:2,6\% & TS:3,9\% & TS:0\% & TS:1,3\% \\
\hline & TS:98\% & TS:85\% & & & $p=0,483$ & $\mathrm{p}=0,610$ \\
\hline \multirow[t]{3}{*}{ Dhillon y cols ${ }^{26}$} & \multirow[t]{3}{*}{-} & \multirow[t]{3}{*}{-} & $\begin{array}{l}\text { TC:0,61 (IC95\%, } \\
0,05-0,49)\end{array}$ & $\begin{array}{l}\text { TC:0,45 } \\
(\mathrm{IC} 95 \%,\end{array}$ & \multirow[t]{3}{*}{-} & \multirow[t]{3}{*}{-} \\
\hline & & & \multirow{2}{*}{$\begin{array}{l}\text { TS 0,20 (IC95\%, } \\
0,00-2,46)\end{array}$} & $0.07-2.20)$ & & \\
\hline & & & & $\begin{array}{l}\text { TS 0,30 } \\
\text { (IC95\%, } \\
0,00-4,46)\end{array}$ & & \\
\hline
\end{tabular}

TC: Técnica convencional. TS: Técnica de Solomon.

Tabla II

SÍNDROME DE TRANSFUSIÓN FETO FETAL, RESULTADOS DEL SEGUIMIENTO DEL NEURODESARROLLO: TÉCNICA CONVENCIONAL VS TÉCNICA DE SOLOMON

\begin{tabular}{lccccc}
\hline Estudio & $\begin{array}{c}\text { Sobrevida sin } \\
\text { deterioro }\end{array}$ & $\begin{array}{c}\text { Alteración } \\
\text { neurodesarrollo }\end{array}$ & $\begin{array}{l}\text { Parálisis } \\
\text { cerebral }\end{array}$ & $\begin{array}{c}\text { Desarrollo } \\
\text { cognitivo }(<85)\end{array}$ & $\begin{array}{c}\text { Desarrollo } \\
\text { motor }(<85)\end{array}$ \\
\hline Van Klink $^{27}$ cols $^{27}$ & TC 68\% & TC 11\% & TC 2\% & TC 6\% & TC 3\% \\
& TS 67\% & TS 9\% & TS 1\% & TS 2\% & TS 8\% \\
& $\mathrm{p}=0,92$ & $\mathrm{p}=0,61$ & $\mathrm{p}=0,58$ & $\mathrm{p}=0,23$ & $\mathrm{p}=0,23$ \\
\hline
\end{tabular}

TC: Técnica convencional. TS: Técnica de Solomon.

\section{DISCUSIÓN}

El síndrome de transfusión feto/fetal es una de las complicaciones del embarazo monocorial biamniótico que causa una alta morbilidad y mortalidad; gracias al advenimiento de la ablación con láser se incrementó la probabilidad de sobrevida de uno o de ambos fetos y ésta se ha convertido en la técnica de elección $(29,30)$, sin embargo, las complicaciones en el neourodesarrollo se siguen presentando y no ha sido posible eliminarlas (31).

Con el tiempo se ha venido ganando experticia en la técnica quirúrgica, en los últimos años ha aparecido una nueva denominada técnica de Solomon, la cual ofrece además del manejo estándar, coagulación adicional del área entre las anastomosis en el ecuador vascular. 
La presente revisión en la literatura buscó comparar estas dos técnicas, la técnica estándar de fotocoagulación secuencial con láser y la técnica de Solomon. El resultado primario fue la incidencia de secuencia anemia policitemia, recurrencia del síndrome de transfusión feto/fetal, mortalidad perinatal, o morbilidad neonatal severa.

Se encontraron 3 estudios primarios todos de alta calidad (18-20), dos tipo cohorte retrospectivos $(24,25)$ y un ensayo clínico aleatorizado abierto multicéntrico con su análisis secundario (22) y un meta análisis (26) de estos tres estudios.

En los estudios de cohorte se sugiere una mejoría en la sobrevida de alguno de los fetos con la técnica de Solomon que al parecer presenta mejores tasa de sobrevida, aunque hay diferencias en los puntos finales, ya que el estudio de Ruano y cols (24) evidencia mejoría en la sobrevida a los 30 días para ambos fetos y no para uno de ellos; el estudio de Baschat (25) la evalúa a los 6 meses. Este hallazgo no fue confirmado por el ensayo clínico y se recomienda en futuros estudios utilizar definiciones estandarizadas para sobrevida y resultado del embarazo.

Respecto a la recurrencia de síndrome de transfusión feto/fetal y secuencia anemia policitemia, el estudio de Ruano y cols (24) no mostró diferencias, esto puede ser por la pobre potencia debido al tamaño de muestra, mientras que el estudio de Baschat y cols (25) mostró diferencias significativas con respecto a estos dos puntos, hallazgo que se corroboró en el ensayo clínico donde se encontraron diferencias estadísticamente significativas, con menor incidencia de recurrencia de síndrome de transfusión feto/fetal y secuencia anemia policitemia sin la presencia de eventos adversos; sin embargo, respecto a la mortalidad y resultados a largo plazo sobre todo en el neourodesarrollo los estudios evaluados no permiten inferir conclusiones, ya que por el tamaño de muestra no hay potencia para esto.

El análisis secundario del ensayo clínico aporta las diferencias estadísticamente significativas encontradas respecto al porcentaje de anastomosis residuales menores en la técnica de Solomon $(32,33)$. Estas comunicaciones residuales pueden ser responsables de complicaciones severas postoperatorias como secuencia anemia policitemia en un $13-16 \%$ o recurrencia del síndrome de transfusión feto/fetal en un 7-14\% $(34,35)$.

\section{CONCLUSIÓN}

La presente revisión evidencia que esta nueva técnica tiene menor incidencia de recurrencia tanto del síndrome de transfusión feto/fetal como de la secuencia anemia policitemia, con lo que se disminuirían los riesgos de repetir procedimientos, disminuyendo morbilidad materna y pérdidas o parto pre término y al parecer mejora la sobrevida de alguno de los gemelos. El tiempo quirúrgico y los eventos adversos fueron similares en ambas técnicas, más sin embargo no se puede concluir que hay mejoría en mortalidad, ya que los estudios no tienen el suficiente poder para determinar esto, por lo tanto en este momento no hay evidencia suficiente para recomendar una técnica sobre la otra y se requieren más estudios aleatorizados para encontrar diferencias respecto a mortalidad, sobrevida y morbilidad a largo plazo, sobre todo alteraciones del neurodesarrollo.

\section{REFERENCIAS}

1. Ville Y, Hecher K, Gagnon A, Sebire N, Hyett J, Nicolaides K. Endoscopic laser coagulation in the management of severe twin-to-twin transfusion syndrome. Br J Obstet Gynaecol 1998;105:446-53.

2. Diehl W, Hecher K, Zikulnig L, Vetter M,Hackeloer BJ. Placental vascular anastomoses visualized during fetoscopic laser surgery in severe mid-trimester twintwin transfusion syndrome. Placenta 2001;22:876-81.

3. Bermudez $\mathrm{C}$, Becerra $\mathrm{CH}$, Bornick $\mathrm{PW}$, Allen $\mathrm{MH}$, Arroyo J, Quintero RA. Placental types and twintwin transfusion syndrome. Am J Obstet Gynecol 2002;187:489-94.

4. Quintero R, Quintero L, Bornick P, Allen M, Johnson $P$. The donor-recipient (D-R) score: in vivo endoscopic evidence to support the hypothesis of a net transfer of blood from donor to recipient in twin-twin transfusion syndrome. Prenat Neonat Med 2000;5:84-91.

5. Lewi L, Deprest J, Hecher K. The vascular anastomoses in monochorionic twin pregnancies and their clinical consequences. Am J Obstet Gynecol 2013;208:19-30.

6. Lewi L, Jani J, Blickstein I, et al. The outcome of monochorionic diamniotic twin gestations in the era of invasive fetal therapy: a prospective cohort study. Am J Obstet Gynecol 2008;199:514e1-8.

7. Ong SSC, Zamora J, Khan KS, et al. Prognosis for the co-twin following single-twin death: a systematic review. BJOG 2006; 113:992-8.

8. Hillman SC, Morris RK, Kilby MD. Co-twin prognosis after single fetal death: a systematic review and metaanalysis. Obstet Gynecol 2011;118:928-40.

9. Quintero RA, Morales WJ, Mendoza G, Allen M, Kalter CS, Giannina G, et al. Selective photocoagulation of placental vessels in twinetwin transfusion syndrome: evolution of a surgical technique. Obstet Gynecol Surv 1998;53:S97e103.

10. De Lia JE, Cruikshank DP, Keye WR Jr. Fetoscopic neodymium: YAG laser occlusion of placental vessels in severe twin-twin transfusion syndrome. Obstet Gynecol 1990; 75:1046-53.

11. Ville Y, Hyett J, Hecher K, Nicolaides K. Preliminary experience with endoscopic laser surgery for severe twin-twin transfusion syndrome. $\mathrm{N}$ Engl $\mathrm{J}$ Med 1995;332:224-7.

12. Quintero RA, Bornick PW, Allen MH, Johson PK. Selective laser photocoagulation of communicating vessels in severe twin-twin transfusion syndrome in women with an anterior placenta. Obstet Gynecol 2001;97:477-81. 
13. Lopriore E, Slaghekke F, Middeldorp JM, Klumper FJ, Oepkes D,. Vandenbussche FP. Residual anastomoses in twin-to-twin transfusion syndrome treated with selective fetoscopic laser surgery: localization, size, and consequences. Am J Obstet Gynecol 2009;201:66.e61-4.

14. Cordero L, Franco A, Joy SD, O'Shaughnessy RW. Monochorionic diamniotic infants without twin-to-twin transfusión syndrome. J Perinatol 2005;25:753-58.

15. Quintero RA. Twin-twin transfusion syndrome. Clin Perinatol 2003;30:591-600.

16. Akkermans J, Peeters SH, Klumper FJ, Lopriore E, Middeldorp JM, Oepkes D. Twenty-Five Years of Fetoscopic Laser Coagulation in Twin-Twin Transfusion Syndrome: A Systematic Review. Fetal Diagn Ther 2015 [Epub ahead of print] PubMed PMID: 26278319

17. Senat MV, Deprest J, Boulvain M, Paupe A, Winer N, Ville $Y$. Endoscopic laser surgery versus serial amnioreduction for severe twin-to-twin transfusion syndrome. N Engl J Med 2004;351:136-44.

18. Moher D, Hopewell S, Schulz KF, et al. CONSORT 2010 Explanation and Elaboration: updated guidelines for reporting parallel group randomised trials, BMJ 2010;340:c869

19. Versión en español: Centro Cochrane Iberoamericano, traductores. Manual Cochrane de Revisiones Sistemáticas de Intervenciones, versión 5.1.0 [actualizada en marzo de 2011] [Internet]. Barcelona: Centro Cochrane Iberoamericano;2012.Disponible en http:// www.cochrane.es/?q=es/node/269

20. Vandenbroucke JP, von Elm E, Altman DG, et al. Mejorar la comunicación de estudios observacionales en epidemiología (STROBE): explicación y elaboración. Gac Sanit 2009. doi: 10.1016/j. gaceta. 2008.12.001

21. Urrutia G, Bonfill X. Declaración PRISMA: una propuesta para mejorar la publicación de revisiones sistemáticas y metaanálisis, Med Clin (Barc) 2010;135(11):507-11.

22. Slaghekke F, Lopriore E, Lewi L, et al. Fetoscopic laser coagulation of the vascular equator versus selective coagulation for twin-to-twin transfusion syndrome: an open-label randomised controlled trial. Lancet 2014;383(9935):2144-51

23. Slaghekke F, Lewi L, Middeldorp JM, et al. Residual anastomoses in twin-twin transfusion syndrome after laser: the Solomon randomized trial. Am J Obstet Gynecol 2014;211:285.e1-7.

24. Ruano R, Rodo C, Peiro JL, et al. Fetoscopic laser ablation of placental anastomoses in twin-twin transfusion syndrome using 'Solomon technique'. Ultrasound ObstetGynecol 2013;42:434-9.
25. Baschat AA, Barber J, Pedersen N, Turan OM, Harman CR. Outcome after fetoscopic selective laser ablation of placental anastomoses vs equatorial laser dichorionization for the treatment of twin-to-twin transfusion syndrome. Am J Obstet Gynecol 2013;209:2348.

26. Dhillon RK, Hillman SC, Morris NRK, Kilby MD. Comparison of Solomon technique against selective laser ablation for Twin-Twin Transfusion Syndrome: a systematic review. Ultrasound Obstet Gynecol 2015;46(5):526-33.

27. Slaghekke F, Lewi L, Middeldorp JM, et al. Residual anastomoses in twin-twin transfusion syndrome after laser: the Solomon randomized trial. Am J Obstet Gynecol 2014;211:285.e1-7.

28. Van Klink JM, Slaghekke F, Balestriero MA, et al. Neurodevelopmental outcome at 2 years in twin-twin transfusion syndrome survivors randomized for the Solomon trial. Am J Obstet Gynecol 2016;214(1):113. e1-7.

29. Chalouhi GE, Essaoui M, Stirnemann J, et al. Laser therapy for twin-to-twin transfusion syndrome (TTTS). Prenat Diagn 2011;31:637-46.

30. Taylor MJ, Shalev E, Tanawattanacharoen S, Jolly M, Kumar S, Weiner E, Cox PM, Fisk NM. Ultrasoundguided umbilical cord occlusion using bipolar diathermy for Stage III/IV twin-twin transfusion syndrome. Prenat Diagn 2002;22:70-6.

31. Roberts D, Neilson JP, Kilby MD, Gates S. Interventions for the treatment of twin-twin transfusion syndrome. The Cochrane database of systematic reviews 2014; 1: Cd002073

32. Roberts D, Gates S, Kilby M, Neilson JP. Interventions for twin - twin transfusion syndrome: a Cochrane review. Ultrasound Obstet Gynecol 2008;31:701e11.

33. Lopriore E, Middeldorp JM, Oepkes D, Klumper FJ, Walther FJ, Vandenbussche FP. Residual anastomoses after fetoscopic laser surgery in twin-to-twin transfusion syndrome: frequency, associated risks and outcome. Placenta 2007;28:204-8.

34. Lewi L, Jani J, Cannie M, et al. Intertwin anastomoses in monochorionic placentas after fetoscopic laser coagulation for twin-to-twin transfusion syndrome: is there more than meets the eye? Am J Obstet Gynecol 2006;194:790-5

35. Robyr R, Lewi L, Salomon LJ, et al. Prevalence and management of late fetal complications following successful selective laser coagulation of chorionic plate anastomoses in twin-to-twin transfusion syndrome. Am J Obstet Gynecol 2006;194:796-803. 